\title{
Induction of non-small cell lung carcinoma apoptosis using soluble RGD-TRAIL by targeting the integrin receptor of tumor cells
}

\author{
RUYONG YAO ${ }^{1}$, AIHUA SUI ${ }^{1}$, ZHENLI WANG ${ }^{2}$, SHIHAI LIU ${ }^{1}$, QUAN ZHOU ${ }^{1}$, \\ XIANGPING LIU $^{1}$ and HAIPING ZHANG ${ }^{3}$ \\ ${ }^{1}$ Central Laboratory, Affiliated Hospital of Qingdao University, Medical College, Qingdao 266003; \\ ${ }^{2}$ Department of Pharmacology, The First Sanatorium of Jinan Military District, Qingdao 266001; \\ ${ }^{3}$ Department of Clinical Laboratory, Affiliated Hospital of Qingdao University, Medical College, Qingdao 266003, P.R. China
}

Received May 4, 2012; Accepted August 29, 2012

DOI: $10.3892 / \mathrm{mmr} .2012 .1071$

\begin{abstract}
The tumor necrosis factor-related apoptosisinducing ligand (TRAIL) is a promising candidate for cancer therapeutics that exhibits the ability to preferentially induce apoptosis in malignant cells. RGD peptides bind to the integrins, $\alpha v \beta 3$ and $\alpha v \beta 5$, which are selectively expressed in tumor neovasculature and at the surface of certain tumor cells. To enhance the antitumor effect, an RGD-TRAIL protein, in which TRAIL was fused with the RGD motif-containing cell adhesive sequence, GRGDNP (gly-arg-gly-asp-asn-pro), was constructed and evaluated for bioactivity. The soluble TRAIL and RGD-TRAIL proteins were expressed in Escherichia coli BL21 (DE3) and purified using a non-denaturing method. The antitumor effect of the purified RGD-TRAIL on cell proliferation was evaluated in vitro using MTT and wound healing assays and cell apoptosis was assessed by Hoechst 33342 staining and PARP expression analysis. The results revealed that RGD-TRAIL inhibited the proliferation of multiple tumor cell lines (A549, NCI-H1299 and HCC827). Western blot analysis demonstrated that the treatment of tumor cells with RGD-TRAIL activated the apoptotic pathway by the cleavage of PARP, in the same way as wild-type TRAIL (wtTRAIL). These results demonstrate that RGD-TRAIL possesses more potent antitumor effects than wtTRAIL and, therefore, merits further investigation in preclinical and clinical studies.
\end{abstract}

\section{Introduction}

Lung cancer is one of the most common causes of cancer-related mortality worldwide and approximately $80 \%$ of all lung cancers are of the non-small cell lung carcinoma (NSCLC) type (1). Despite continuous efforts to improve

Correspondence to: Haiping Zhang, Department of Clinical Laboratory, Affiliated Hospital of Qingdao University, Medical College, 16 Jiangsu Road, Qingdao 266003, P.R. China

E-mail: zhp0122@yahoo.cn

Key words: lung tumor, antitumor, integrin, tumor necrosis factor-related apoptosis-inducing ligand conventional cancer treatments, the long-term outcome for many patients treated with surgery, chemotherapy and/or radiotherapy remains suboptimal. The standard treatment of these tumors has only a $20-30 \%$ positive clinical response due to the resistance of the tumors to therapeutic drugs (2). Therefore, in order to develop new therapeutic strategies to improve the treatment of NSCLC, it is important to understand the molecular mechanisms involved in the resistance of the cells to death.

The Apo2 ligand or tumor necrosis factor (TNF)related apoptosis-inducing ligand (Apo2L/TRAIL) was identified and cloned by its sequence homology to TNF and CD95L $(3,4)$. As a member of the TNF family, TRAIL specifically activates extrinsic apoptotic pathways in cancer cells by binding to death receptors. TRAIL is a promising candidate for cancer therapeutics that exhibits the ability to preferentially induce apoptosis in malignant cells (5-7). Significantly, TRAIL selectively promotes the apoptosis of tumor cells but has no effect on normal human reproductive tract cells, including those in the endometrium, ovary, cervix and fallopian tube (8). While many studies have shown TRAIL to be a potent inducer of apoptosis in a wide variety of human and mouse tumor cell lines, many primary tumors are resistant to TRAIL-induced apoptosis and several mechanisms underlying the TRAIL sensitivity/resistance have been proposed (9).

The synthetic integrin-binding peptide, GRGDNP (glyarg-gly-asp-asn-pro), is an integrin-recognition motif found in many ligands. The integrins, $\alpha v \beta 3$ and $\alpha v \beta 5$, serve as receptors for a variety of extracellular matrix proteins having the exposed RGD peptide sequence (10). Integrins $\alpha v \beta 3$ and $\alpha v \beta 5$ are overexpressed in multiple tumor cell types and in the activated endothelial cells of tumor neovasculature (11-13). The highly restricted expression of integrins $\alpha v \beta 3$ and $\alpha v \beta 5$ correlates well with tumor growth, progression, invasion and metastasis. When coupled to an anticancer drug, the RGD peptide may enable targeting of the drug and enhance its antitumor efficacy. Moreover, RGD-containing peptides are able to directly induce apoptosis by triggering conformational changes that promote pro-caspase-3 autoprocessing and activation (14).

In this study, the TRAIL mutant, RGD-TRAIL, was constructed by inserting a GRGDNP peptide before wild-type 
TRAIL (wtTRAIL). The ability of RGD-TRAIL to mediate cancer-selective cell inhibition and its targeted antitumor efficacy in three lung tumor cell lines (A549, NCI-H1299 and HCC827) were assessed. Moreover, the mechanism of the RGD-TRAIL-induced apoptosis was investigated.

\section{Materials and methods}

Cell line and culture. The human lung cancer cell lines, A549, NCI-H1299 and HCC827, and the normal human lung cell line, WI-38, were purchased from the Cell Bank of the Chinese Academy of Sciences. All cell lines were maintained in RPMI-1640 medium supplemented with $10 \%$ fetal bovine serum (FBS; Gibco-BRL, Carlsbad, CA, USA) and $1 \%$ $\mathrm{K}$-glutamine/antimycotic solution. The cells were grown at $37^{\circ} \mathrm{C}$ in a humidified atmosphere containing $5 \% \mathrm{CO}_{2}$.

Construction of expression vectors for TRAIL and mutant RGD-TRAIL. The whole cDNA coding region of the TRAIL gene was cloned from the WI-38 cell line and used as the template for an overlapping polymerase chain reaction (PCR). Three primers for PCR were designed as follows based on the TRAIL sequence and the pET-28a $(+)$ vector: P1, 5'-CGGATC CGTGAGAGAAAGAGGTCCTCAGAGAGT-3'; P2, 5'-CGT CGACTTAGCCAACTAAAAAGGCCCCGAA-3'; and P3, 5'-CGGATCCGGGCGCGGCGATAACCCTGTGAGAGA AAGAGGTCCTCAGAGAGT-3'. P1: the underlined sequence is BamHI; P2 is SalI; P3 is Bam HI. P1/P3 and P2 had BamHI and SalI recognition sites, respectively, for directed cloning into the vector. Using $\mathrm{P} 1 / \mathrm{P} 2$ and $\mathrm{P} 2 / \mathrm{P} 3$ primers for $\mathrm{PCR}$, agarose gel purification was performed for the two products to obtain the fragments encoding TRAIL and RGD-TRAIL, respectively. The fragments were digested with BamHI and SalI and then inserted inframe into the $\mathrm{pET}-28 \mathrm{a}(+)$ expression vector to construct the pET28-TRAIL and pET28-RGD-TRAIL plasmids. The plasmids were confirmed by nucleotide sequencing. The pET-28a(+) expression vector contains a $6 \mathrm{xHis}$ tag to allow immobilized metal ion affinity purification.

Expression and purification of wTTRAIL and RGD-TRAIL proteins. The plasmids were used to transform Escherichia coli (E. coli) strain BL21 (DE3) (resistant to $15 \mu \mathrm{g} / \mathrm{ml}$ kanamycin) competent cells. Positive clones were confirmed by restriction analysis. BL21 (DE3) competent cells were transformed with the RGD-TRAIL construct and the expression of recombinant RGD-TRAIL was determined following induction with $1 \mathrm{mM}$ IPTG. To purify the RGD-TRAIL protein, cultures were grown in shaker flasks at $22^{\circ} \mathrm{C}$ for $16 \mathrm{~h}$ and, following induction, the cells were pelleted. The bacterial pellets were resuspended in lysis buffer $(50 \mathrm{mM}$ Tris $\mathrm{pH} 7.4,500 \mathrm{mM} \mathrm{NaCl}$ and $0.1 \%$ Triton X-100) and sonicated in ice-water baths (15 sec bursts, $30 \mathrm{sec}$ cooling at 200-300 W) for 50 cycles. The lysate was centrifuged at $10,000 \mathrm{x} \mathrm{g}$ for $30 \mathrm{~min}$. The supernatant was mixed with Ni-NTA agarose beads for $1 \mathrm{~h}$ to allow the protein to bind to the beads. After binding, the beads were packed into a column and washed with $100 \mathrm{ml}$ Tris-buffered saline. The RGD-TRAIL protein was eluted with $250 \mathrm{mM}$ imidazole in Tris-buffered saline and dialyzed against phosphate-buffered saline (PBS) followed by DMEM. The protein was passed through a $0.2 \mu \mathrm{m}$ filter, quantified, aliquoted and stored in ice at $-80^{\circ} \mathrm{C}$. Recombinant TRAIL was purified similarly. Protein purity and identity were determined by sodium dodecyl sulfate-polyacrylamide gel electrophoresis (SDS-PAGE) and western blot analysis.

Immunofluorescence analyses. A549 cells were grown on chamber slides (BD Falcon ${ }^{\mathrm{TM}}$; BD Biosciences, San Jose, CA, USA) fixed with $2 \%$ paraformaldehyde, permeabilized by $0.1 \%$ Triton X-100 and then incubated with the primary antibodies anti-rabbit TRAIL and TRITC-conjugated goat anti-rabbit IgG (Molecular Probes, Eugene, OR, USA). Sections were stained for 5 min with DAPI, washed an additional three times with PBS and then visualized under a Leica DMI4000B fluorescence microscope (Leica Microsystems GmbH Wetzlar, Germany).

Cell viability assay. The cells were plated in 96-well plates and treated with the RGD-TRAIL and wtTRAIL proteins at the concentrations designated in Fig. 2. At the indicated times, the medium was removed and fresh medium containing 3-(4,5-dimethylthiazol-2-yl)-2,5-diphenyltetrazolium bromide (MTT, $0.5 \mathrm{mg} / \mathrm{ml}$; Sigma, St. Louis, MO, USA) was added to each well. The cells were incubated at $37^{\circ} \mathrm{C}$ for $4 \mathrm{~h}$, then the medium was removed and $150 \mu 1$ solubilization solution (DMSO) was added and mixed in thoroughly. The absorbance of the plates at $490 \mathrm{~nm}$ was read using a Safire II spectrometer reader (Tecan Trading AG, Männedorf, Switzerland).

Hoechst 33342 staining. The nuclear DNA in the treated cells contained in 24-well plates was visualized by staining with the DNA-specific dye Hoechst 33342 at a final concentration of $5 \mu \mathrm{g} / \mathrm{ml}$. The cells were observed immediately with filters for blue fluorescence.

Motility assays. For the wound healing assay, A549 cells plated on fibronectin-coated 24-well plates were grown to confluence. The cells were then scratched with the side corner of a cell lifter, washed three times with DMEM and incubated in DMEM supplemented with 2\% FBS for $11 \mathrm{~h}$. Images of the same fields were captured with a Leica microscope following the scratch and at the end of the incubation time. The covered area in each sample was calculated and compared with the covered area in the control sample. The results presented are an average of three repetitions and the statistical significance was determined by the Student's t-test.

Western blot analysis. Cell lines were grown on $10-\mathrm{cm}$ plates and protein extracts were prepared using a RIPA buffer containing a cocktail of protease inhibitors. A 50- $\mu \mathrm{g}$ sample of the protein was applied to $15 \%$ SDS-PAGE and transferred to nitrocellulose membranes. The membranes were probed with polyclonal or monoclonal antibodies to TRAIL and cleaved PARP, a general marker of apoptosis.

Statistical analysis. All values are expressed as the means $\pm \mathrm{SD}$, and statistical analysis of the results was carried out by one-way analysis of variance followed by Duncan's new multiple range method or Newman-Keuls test. A value of $p<0.05$ was considered to indicate a statistically significant result. 


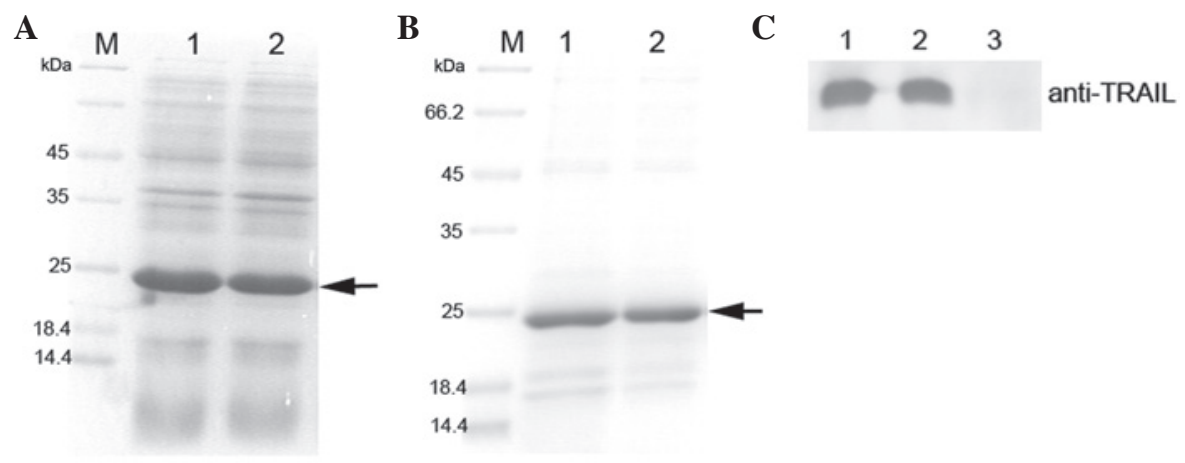

Figure 1. Expression, purification, and identification of RGD-TRAIL and wtTRAIL. (A) SDS-PAGE gel analysis of TRAIL. M, protein marker; lane 1, pET-28a-TRAIL, whole cell lysate induced by IPTG; lane 2, pET-28a-RGD-TRAIL, whole cell lysate induced by IPTG. (B) SDS-PAGE gel analysis of TRAIL. M, protein marker; lane 1, purified wtTRAIL; lane 2, purified RGD-TRAIL. (C) Western blot analysis of TRAIL protein. Lane 1, wtTRAIL; lane 2, RGD-TRAIL; lane 3, blank control without IPTG induction. TRAIL, tumor necrosis factor-related apoptosis-inducing ligand; SDS-PAGE, sodium dodecyl sulfate-polyacrylamide gel electrophoresis.

A

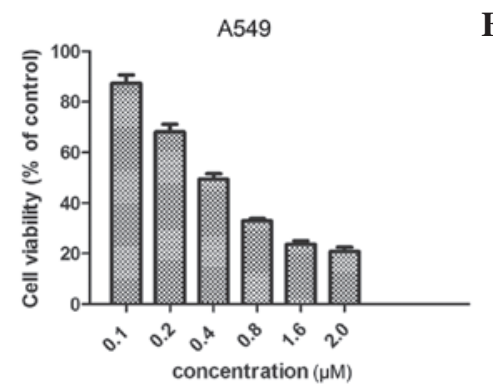

D

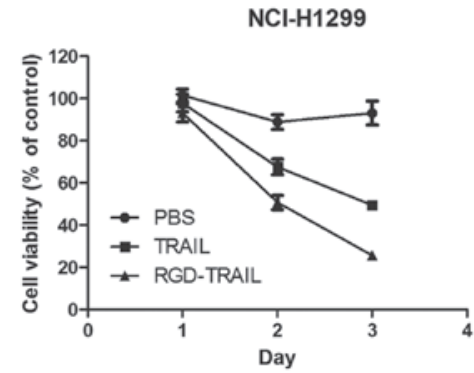

B

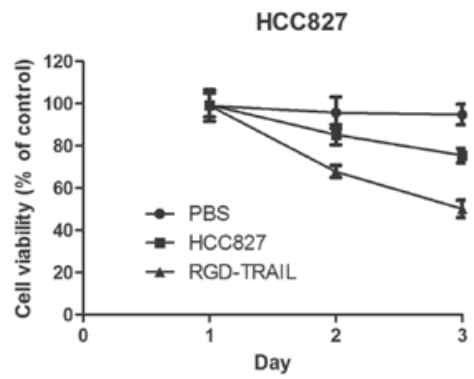

$\mathbf{E}$

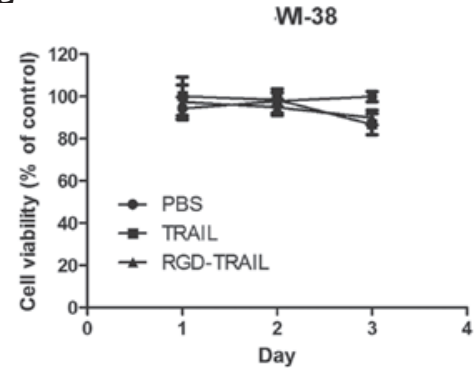

Figure 2. Effect of RGD-TRAIL on the viability of tumor cells. (A) Tumor cells were treated with RGD-TRAIL for $48 \mathrm{~h}$, at concentrations of $0.1,0.2,0.4,0.8$, 1.6 and $2 \mu \mathrm{M}$. Cell viability was determined by the MTT assay. (B-E) Tumor and normal cells were treated with RGD-TRAIL, TRAIL and PBS at a concentration of $0.8 \mu \mathrm{M}$. On days 1,2 and 3 post-treatment, cell viability was determined by the MTT assay. p<0.05 (RGD-TRAIL vs. wtTRAIL). TRAIL, tumor necrosis factor-related apoptosis-inducing ligand; MTT, 3-(4,5-dimethylthiazol-2-yl)-2,5-diphenyltetrazolium bromide; PBS, phosphate-buffered saline.

\section{Results}

Expression and purification of the recombinant wild-type (wt) and RGD-TRAIL proteins. By means of overlapping PCR, an RGD-TRAIL mutant was created. The RGD-TRAIL was cloned into the pET-28a(+) vector and transformed into DH5a competent cells. The expected mutation was confirmed by DNA sequencing. The transformed BL21 (DE3) cells were inoculated into Overnight Express Instant LB medium at $22^{\circ} \mathrm{C}$ for $16 \mathrm{~h}$ following the addition of $1 \mathrm{mM}$ IPTG. After induction, the cells were dissolved in SDS-PAGE sample buffer and subjected to SDS-PAGE. The results revealed that the recombinant wtTRAIL and RGD-TRAIL proteins were efficiently expressed, with yields accounting for $30 \%$ of total bacterial proteins (Fig. 1A).

To investigate the apoptotic activities of the recombinant wtTRAIL and RGD-TRAIL proteins, we purified TRAIL protein from E. coli using the non-denaturing method as described in Materials and methods. SDS-PAGE gel analysis confirmed that the recombinant wtTRAIL and RGD-TRAIL proteins were purified, respectively (Fig. 1B). Due to the removal of the signal peptides of TRAIL, the soluble protein activities of the purified target proteins were improved. We obtained $\sim 1.0 \mathrm{mg}$ wtTRAIL and RGD-TRAIL, respectively, per liter of bacterial culture. Western blot analysis confirmed that the RGD-TRAIL and wtTRAIL proteins were recognized by the anti-TRAIL monoclonal antibody (Fig. 1C).

Inhibition of cell viability is induced by RGD-TRAIL in tumor cells but not in normal lung cells. To assess the cytopathic effect of RGD-TRAIL, an MTT assay was performed to examine cell viability. A549 cells were plated on 96-well plates and treated with RGD-TRAIL at various concentrations. As shown 


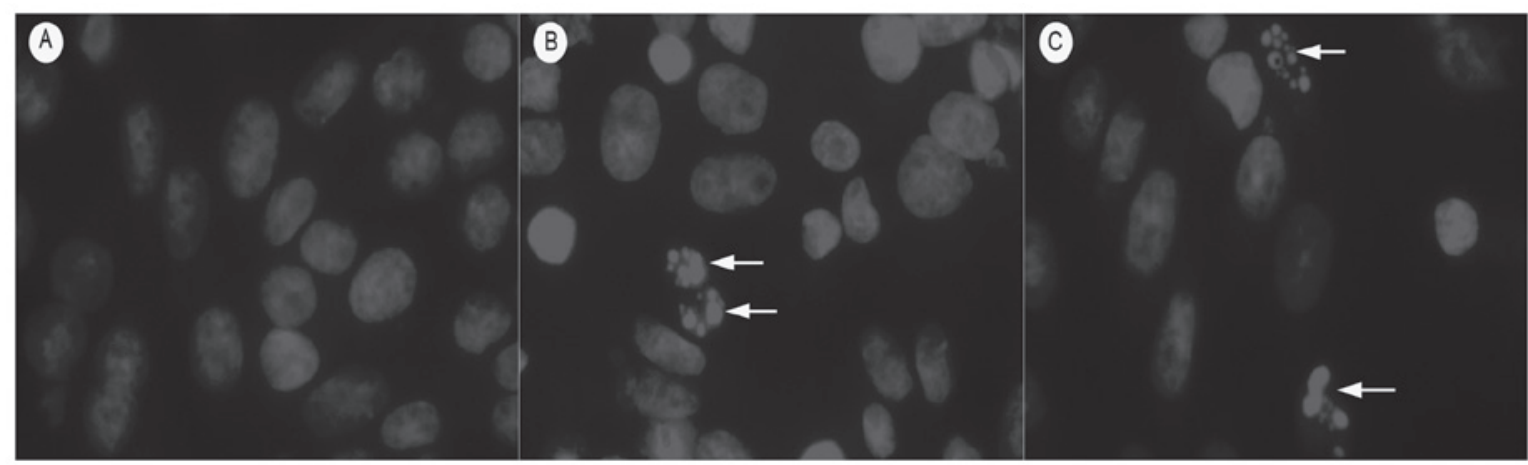

Figure 3. RGD-TRAIL-infected A549 cells undergo apoptotic cell death. Nuclear fragmentation was examined by Hoechst 33342 nuclear staining in A549 cells treated with (B) wtTRAIL or (C) RGD-TRAIL. (A) PBS treatment was used as a control. TRAIL, tumor necrosis factor-related apoptosis-inducing ligand.

in Fig. 2A, the treatment of the A549 cells with RGD-TRAIL resulted in a dose-dependent cytopathic effect at $48 \mathrm{~h}$. As expected, the tumor cells were inhibited by RGD-TRAIL more powerfully than by wtTRAIL. The treatment of the HCC827, A549 and NCI-H1299 tumor cells with RGD-TRAIL resulted in a significant decrease in cell viability compared with the cells treated with wtTRAIL (Fig. 2B-D). In contrast, normal cells (WI-38) treated with either RGD-TRAIL or wtTRAIL did not demonstrate any change in cell viability (Fig. 2E).

RGD-TRAIL induces apoptosis in tumor cells. Apoptotic cells demonstrating nuclear condensation and DNA fragmentation were detected by Hoechst 33342 staining and fluorescence microscopy. As illustrated in Fig. 3, the lung cancer cells incubated with TRAIL and RGD-TRAIL for $24 \mathrm{~h}$ exhibited many more cells with condensed and fragmented nuclei than those treated with PBS alone (Fig. 3).

To further investigate the effect of the TRAIL treatment on A549 cells, we subjected the cells to the scratch wound assay of in vitro repair. The same fields of confluent cells were pictured immediately following the scratch (time 0 ) and following $24 \mathrm{~h}$ of incubation. We examined the migration rates of the A549 cells treated with PBS, TRAIL and RGD-TRAIL proteins. The RGD-TRAIL protein treatment lowered the level of the wound closure to $70 \%$ of the control sample (Fig. 4).

Previous reports have demonstrated that TRAIL-induced cell death occurs through an apoptotic mechanism characterized by the activation of a cascade of intracellular proteases and the cleavage of numerous intracellular proteins $(15,16)$. To confirm that the tumor cell death following wtTRAIL and RGD-TRAIL treatment was mediated through an apoptotic mechanism, nuclear fragmentation and cellular protein cleavage were examined. A549 cells were incubated with RGD-TRAIL, wtTRAIL and PBS for $24 \mathrm{~h}$, cell lysates were prepared at various times following incubation and the cellular proteins were separated by SDS-PAGE for western blot analysis of the cleavage of PARP, a substrate for caspase-3 (Fig. 5). These results demonstrated that treatment with the TRAIL gene effectively elicited apoptosis in the cultured lung tumor cells.

\section{Discussion}

The specific induction of apoptosis in tumor cells is a promising therapeutic approach for cancer. In this study, we showed that the RGD-TRAIL protein selectively localized in tumors and resulted in the apoptosis of the tumor cells. This treatment combines the advantages of RGD and the potent antitumor effects of TRAIL. The TRAIL protein in various forms has been demonstrated to induce apoptosis in many cancer cells in vitro and to inhibit the growth of many tumors in rodent models. The TRAIL protein acts by activating the cell surface receptors DR4 and DR5 $(17,18)$. In our study, the RGD-TRAIL protein mediated the inhibition of tumor growth and induced A549 cell apoptosis in vitro.

The combination of direct cancer-specific apoptosis induction and indirect antitumor properties makes TRAIL an ideal cancer therapeutic. A significant feature of TRAIL is its non-toxicity toward normal cells that may be exploited to target the expression of TRAIL to cancer and normal cells, the latter serving as a reservoir for the continuous secretion of TRAIL protein, thereby exerting profound bystander antitumor effects. It is imperative that TRAIL be delivered by a vector system that employs a universal entry system into the cells and is also shielded from immunologic clearance from the circulation. In the present study, an RGD-TRAIL mutant was constructed and its ability to mediate cancer-selective cell inhibition and to target tumor cells was assessed. The recombinant protein was efficiently expressed in E. coli strain BL21 (DE3). The integrins, $\alpha v \beta 3$ and $\alpha v \beta 5$, are receptors for the RGD tripeptide sequence and play a significant role in tumor progression, invasion and metastasis (19). In the present study, a significant cytopathic effect was observed by the MTT assay in all tumor cell lines treated with RGD-TRAIL, compared with wtTRAIL. This cytopathic effect was timeand dosage-dependent. The superior antitumoral efficacy of RGD-TRAIL may be due to the interaction of the RGD sequence with integrins $\alpha v \beta 3$ and $\alpha v \beta 5$ in the tumor cell membrane. These results suggest that the insertion of Gly into wtTRAIL does not disrupt its biological function and selective inhibitory effects in tumor cells and that RGD-TRAIL inhibits the growth of tumor cells more effectively than wtTRAIL.

As shown in Fig. 3, RGD-TRAIL elicited typical apoptotic morphological changes in the A549 tumor cells, including chromatin condensation and apoptotic bodies. By contrast, no significant morphological changes were found in the WI-38 cell control group. Moreover, statistical analysis revealed that the apoptosis-inducing activity of RGD-TRAIL in the 


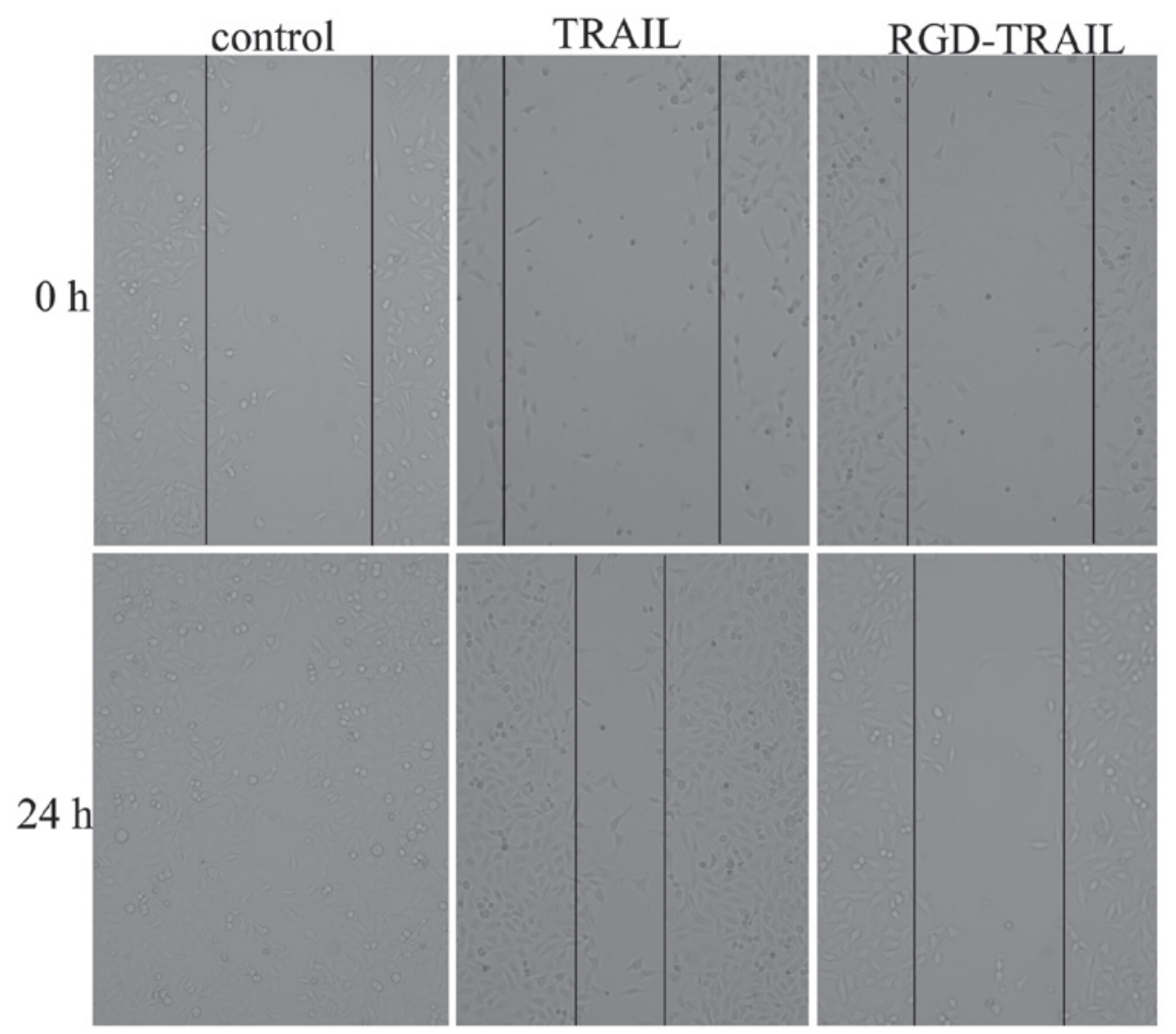

Figure 4. TRAIL protein inhibits the migration rate of A549 cells in the wound healing assay. The migration rate of the A549 cells was measured in the presence of RGD-TRAIL, TRAIL or PBS. Representative phase-contrast images of the cells with the various treatment agents at time $0 \mathrm{~h}$ (immediately following the scratch) and $24 \mathrm{~h}$ are shown. TRAIL, tumor necrosis factor-related apoptosis-inducing ligand; PBS, phosphate-buffered saline.

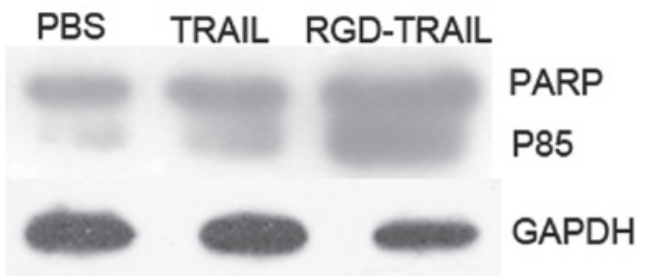

Figure 5. Cleavage of PARP from 118 to $85 \mathrm{kDa}$ (P85) occurs during apoptotic cell death. Cell lysates were prepared following induction for $48 \mathrm{~h}$ and cellular proteins were determined by western blot analysis.

tumor cells was much stronger than that of wtTRAIL. An explanation for the difference is that RGD-TRAIL enhanced the antitumor potency of wtTRAIL. A number of studies have sought to identify the molecules involved in the TRAILinduced apoptotic signaling in tumor cells. It is evident, based on existing data, that the tumor-specific apoptotic activity of TRAIL involves multiple pathways. These pathways appear to be regulated only in tumor cells. Our results revealed that the activation of PARP occurred in tumor cells treated with RGD-TRAIL and wtTRAIL but not in untreated control cells (Fig. 5). These results demonstrate that RGD-TRAIL induced apoptosis and activated PARP.

In conclusion, we have successfully prepared RGD-TRAIL, and confirmed that it has much stronger anticancer efficacy than TRAIL or PBS. This study provides an effective model drug for subsequent studies concerning lung cancer treatment. The fusion of RGD with TRAIL to construct RGD-TRAIL not only does not disrupt the biological function of TRAIL but also enhances its growth suppression and apoptosis-inducing effects in multiple human tumor cell lines, but not in normal cells (WI-38). Although the antitumor mechanism of RGD-TRAIL requires further elucidation, the preliminary data from this study indicate that the RGD-modification of TRAIL is an effective approach to enhance the antitumoral potency of TRAIL.

\section{Acknowledgements}

This study is part of a project financed by a grant from Shandong Tackle Key Problems in Science and Technology (2010GSF10245) and Shandong Excellent Young Scientist Research Award Fund Project (BS2010YY013).

\section{References}

1. Travis WD: Pathology of lung cancer. Clin Chest Med 23: 65-81, 2002.

2. Zaba $\mathrm{O}$, Grohe $\mathrm{C}$ and Merk J: Novel therapies in non-small cell lung cancer. Minerva Chir 66: 235-244, 2011.

3. Call JA, Eckhardt SG and Camidge DR: Targeted manipulation of apoptosis in cancer treatment. Lancet Oncol 9: 1002-1011, 2008. 
4. Walczak H, Miller RE, Ariail K, et al: Tumoricidal activity of tumor necrosis factor-related apoptosis-inducing ligand in vivo. Nat Med 5: 157-163, 1999.

5. Amm HM, Oliver PG, Lee CH, Li Y and Buchsbaum DJ: Combined modality therapy with TRAIL or agonistic death receptor antibodies. Cancer Biol Ther 11: 431-449, 2011.

6. Szliszka E and Krol W: The role of dietary polyphenols in tumor necrosis factor-related apoptosis inducing ligand (TRAIL)induced apoptosis for cancer chemoprevention. Eur J Cancer Prev 20: 63-69, 2011.

7. Voelkel-Johnson C: TRAIL-mediated signaling in prostate, bladder and renal cancer. Nat Rev Urol 8: 417-427, 2011.

8. Sadarangani A, Kato S, Espinoza N, et al: TRAIL mediates apoptosis in cancerous but not normal primary cultured cells of the human reproductive tract. Apoptosis 12: 73-85, 2007.

9. Dyer MJ, MacFarlane M and Cohen GM: Barriers to effective TRAIL-targeted therapy of malignancy. J Clin Oncol 25: 4505-4506, 2007.

10. Liu S: Radiolabeled multimeric cyclic RGD peptides as integrin alphavbeta 3 targeted radiotracers for tumor imaging. Mol Pharm 3: 472-487, 2006.

11. Falcioni R, Cimino L, Gentileschi MP, et al: Expression of beta 1, beta 3 , beta 4 , and beta 5 integrins by human lung carcinoma cells of different histotypes. Exp Cell Res 210: 113-122, 1994.

12. Kumar CC: Integrin alpha $v$ beta 3 as a therapeutic target for blocking tumor-induced angiogenesis. Curr Drug Targets 4: 123-131, 2003.
13. Zitzmann S, Ehemann V and Schwab M: Arginine-glycineaspartic acid (RGD)-peptide binds to both tumor and tumor-endothelial cells in vivo. Cancer Res 62: 5139-5143, 2002.

14. Buckley CD, Pilling D, Henriquez NV, et al: RGD peptides induce apoptosis by direct caspase-3 activation. Nature 397 : 534-539, 1999.

15. Griffith TS, Chin WA, Jackson GC, Lynch DH and Kubin MZ: Intracellular regulation of TRAIL-induced apoptosis in human melanoma cells. J Immunol 161: 2833-2840, 1998.

16. Schneider P, Thome M, Burns K, et al: TRAIL receptors 1 (DR4) and 2 (DR5) signal FADD-dependent apoptosis and activate NF-kappaB. Immunity 7: 831-836, 1997.

17. Pan G, O'Rourke K, Chinnaiyan AM, et al: The receptor for the cytotoxic ligand TRAIL. Science 276: 111-113, 1997.

18. Walczak H, Degli-Esposti MA, Johnson RS, et al: TRAIL-R2 a novel apoptosis-mediating receptor for TRAIL. EMBO J 16 5386-5397, 1997.

19. Reardon DA, Neyns B, Weller M, Tonn JC, Nabors LB and Stupp R: Cilengitide: an RGD pentapeptide $\alpha v \beta 3$ and $\alpha v \beta 5$ integrin inhibitor in development for glioblastoma and other malignancies. Future Oncol 7: 339-354, 2011. 